PROCEEDINGS OF THE

AMERICAN MATHEMATICAL SOCIETY

Volume 134, Number 4, Pages 1161-1165

S 0002-9939(05)08083-4

Article electronically published on October 5, 2005

\title{
SUR LES OPÉRATEURS DE DUNFORD-PETTIS POSITIFS QUI SONT FAIBLEMENT COMPACTS
}

\author{
BELMESNAOUI AQZZOUZ, REDOUANE NOUIRA, AND LARBI ZRAOULA
}

(Communicated by Joseph A. Ball)

\begin{abstract}
RÉsumÉ. Nous donnons des conditions nécessaires et suffisantes pour que tout opérateur de Dunford-Pettis positif sur un treillis de Banach, soit faiblement compact et nous déduisons quelques conséquences.

ABSTRACT. We give necessary and sufficient conditions so that every positive Dunford-Pettis operator on a Banach lattice be weakly compact, and we deduce some consequences.
\end{abstract}

\section{INTRODUCTION ET RAPPELS}

Le problème de domination pour les opérateurs faiblement compacts positifs sur les treillis de Banach a été étudié par Aliprantis et Burkinshaw dans [3] et Wickstead dans [9]. Il s'agit de donner des conditions sur les treillis de Banach $E$ et $F$ pour qu'un opérateur positif $S$ de $E$ vers $F$ dominé par un opérateur positif faiblement compact $T$ soit aussi faiblement compact. Aliprantis et Burkinshaw ont montré que si $E$ et $F$ sont des treillis de Banach tel que la norme de $E^{\prime}$ ou celle de $F$ est continue pour l'ordre, alors pour tous opérateurs $S$ et $T$ de $E$ vers $F$ tels que $0 \leq S \leq T$ et $T$ faiblement compact, l'opérateur $S$ est faiblement compact [2, Théorème 7]. Pendant la même année et par des méthodes différentes, Wickstead 9] a établi le même résultat.

D'autre part, le module d'un opérateur faiblement compact n'existe pas toujours, et si ce module existe il n'est pas nécessairement faiblement compact. Dans [2, Théorème 9], Aliprantis et Burkinshaw ont montré que tout opérateur faiblement compact d'un AL-espace vers un KB-espace (i.e. la norme de son dual topologique est continue pour l'ordre) admet un module faiblement compact. Ensuite, Chen et Wickstead [7, Théorème 2.7(2)] ont établi l'existence d'un treillis de Banach complet pour l'ordre $E$ et de deux opérateurs compacts $S$ et $T$ sur $E$ tels que $S,-S \leq T$ mais le module $|S|$ de $S$ n'est pas faiblement compact. Aussi, ils ont donné des conditions nécessaires et suffisantes pour que tout opérateur faiblement compact d'un AL-espace vers un treillis de Banach (resp. d'un treillis de Banach vers un AM-espace) admet un module faiblement compact [7, Théorème 2.3 et Théorème 2.4]. Enfin, rappelons qu'Aliprantis et Burkinshaw ont montré dans 3 , Théorème 2.8] que si $E$ est un treillis de Banach tel que la norme de $E$ ou celle de $E^{\prime}$ est

Received by the editors October 8, 2004 and, in revised form, November 10, 2004.

2000 Mathematics Subject Classification. Primary 46B40, 47H07.

(C)2005 American Mathematical Society 
continue pour l'ordre, alors tout opérateur de Dunford-Pettis positif de $E$ vers lui même est faiblement compact.

L'objectif de ce papier est d'établir que tout opérateur de Dunford-Pettis positif sur un treillis de Banach $E$ est faiblement compact si, et seulement si, la norme de $E^{\prime}$ est continue pour l'ordre. Ensuite, nous donnons quelques conséquences et remarques.

Avant d'établir le résultat principal de ce papier, nous rappelons ci-dessous quelques définitions. Un élément non nul d'un treillis vectoriel $E$ est dit discret si l'idéal d'ordre engendré par $u$ coïncide avec le sous-treillis vectoriel engendré par $u$. Le treillis vectoriel $E$ est dit discret s'il admet un système disjoint complet d'éléments discrets.

Un treillis de Banach est un espace de Banach $(E,\|\|)$ tel que $E$ est un treillis vectoriel et la norme de $E$ est de treillis (i.e. pour tous $x, y \in E$ tels que $|x| \leq|y|$, on a $\|x\| \leq\|y\|)$. La norme \|\| d'un treillis de Banach $E$ est dite continue pour l'ordre si pour toute suite généralisée $\left(x_{\alpha}\right)$ telle que $x_{\alpha} \downarrow 0$ dans $E$, la suite $\left(x_{\alpha}\right)$ converge vers 0 pour la norme \|\| , où la notation $x_{\alpha} \downarrow 0$ signifie que la suite $\left(x_{\alpha}\right)$ est décroissante et $\inf \left(x_{\alpha}\right)=0$. Pour plus d'informations sur ces notions, nous renvoyons le lecteur au livre d'Aliprantis et Burkinshaw [5].

\section{LE RESULTAT PRINCIPAL}

Un opérateur entre des treillis de Banach est une application linéaire continue. Si $E$ et $F$ sont des treillis de Banach, l'opérateur $T: E \longrightarrow F$ est dit positif si $T(x) \geq 0$ lorsque $x \geq 0$. Notons que toute application linéaire positive d'un treillis de Banach vers un treillis vectoriel normé est nécessairement continue.

Un opérateur $T: E \longrightarrow F$ entre des espace de Banach est dit de Dunford-Pettis si l'image de toute partie faiblement compacte de $E$ est une partie compacte dans F.

Rappelons qu'un espace de Banach $E$ admet la propriété de Dunford-Pettis si toute partie faiblement compacte de $E$ est compacte. Comme exemples, les treillis de Banach $L^{1}([0,1])$ et $l^{\infty}$ ont la propriété de Dunford-Pettis.

Il est clair qu'un espace de Banach $E$ admet la propriété de Dunford-Pettis si, et seulement si, tout opérateur faiblement compact de domaine $E$ est de DunfordPettis.

Réciproquement, l'opérateur identité $I d_{l^{1}}: l^{1} \longrightarrow l^{1}$ est de Dunford-Pettis et positif mais il n'est pas faiblement compact. Pour plus de détails sur les différentes notions des opérateurs positifs sur les treillis de Banach, nous renvoyons le lecteur au livre d'Aliprantis et Burkinshaw [4].

La preuve de notre résultat principal utilisera le lemme suivant:

Lemme 2.1. Soient $E$ un treillis de Banach et $S, T$ deux opérateurs de $E$ vers $E$ tels que $0 \leq S \leq T$ avec $T$ de Dunford-Pettis. Si la norme du dual topologique $E^{\prime}$ de $E$ est continue pour l'ordre, alors pour tout $\varepsilon>0$, il existe un $y \in E^{+}$tel que $S\left(B_{E} \cap E^{+}\right) \subset \varepsilon B_{E}+S([0, y])$, où $B_{E}$ est la boule unité de $E$ et $E^{+}=\{x \in E$ : $0 \leq x\}$.

Preuve. Elle résulte de la démonstration du Théorème 2.7 et du Théorème 2.8 de [3.

Nous établissons maintenant notre résultat principal. Il donne des conditions nécessaires et suffisantes pour que tout opérateur de Dunford-Pettis positif soit faiblement compact. 
Théorème 2.2. Soit $E$ un treillis de Banach. Alors les assertions suivantes sont équivalentes:

1) Tout opérateur de Dunford-Pettis positif de E vers $E$ est faiblement compact.

2) Pour tout opérateur de Dunford-Pettis positif $T$ de $E$ vers E, l'opérateur $T^{2}$ est compact.

3) La norme du treillis de Banach $E^{\prime}$ est continue pour l'ordre.

4) Pour tous opérateurs $S$ et $T$ de $E$ vers $E$ tels que $0 \leq S \leq T$ et $T$ de Dunford-Pettis, l'opérateur $S$ est faiblement compact.

Preuve. Pour l'implication 1) $\Longrightarrow$ 2), soit $T$ un opérateur de Dunford-Pettis positif de $E$ vers $E$. Il est donc faiblement compact, i.e. $T\left(B_{E}\right)$ est relativement compact pour la topologie $\sigma\left(E, E^{\prime}\right)$ dans $E$, où $B_{E}$ est la boule unité de $E$. Comme $T$ est de Dunford-Pettis, alors $T^{2}\left(B_{E}\right)$ est relativement compact dans $E$.

L'implication 2) $\Longrightarrow$ 3). Supposons que la norme du treillis de Banach $E^{\prime}$ n'est pas continue pour l'ordre. Alors il résulte de la démonstration du Théorème 1 de [10] que $E$ contient un sous-treillis vectoriel isomorphe (en ordre) au treillis de Banach $l^{1}$, et il existe aussi une projection positive $P: E \longrightarrow l^{1}$. Il est clair que l'opérateur $P$ est de Dunford-Pettis, mais l'opérateur $P^{2}=P$ n'est pas compact. Sinon la restriction de $P$ à $l^{1}$ qui est l'application identité $I d_{l^{1}}: l^{1} \longrightarrow l^{1}$ sera compact, d'où la contradiction. Il s'ensuit alors que la norme de $E^{\prime}$ est continue pour l'ordre.

Pour l'implication 3$) \Longrightarrow 4$ ), d'après le Lemme 2.1, pour tout $\varepsilon>0$, il existe un $y \in E^{+}$tel que

$$
S\left(B_{E} \cap E^{+}\right) \subset \varepsilon B_{E}+S([0, y]),
$$

où $B_{E}$ est la boule unité de $E$. Comme $S([0, y])$ est faiblement compact [3, Théorème 1.2] alors $S\left(B_{E} \cap E^{+}\right)$est faiblement précompact. Pour montrer qu'il est faiblement relativement compact, il suffit de montrer que ${\overline{S\left(B_{E} \cap E^{+}\right)}}^{\sigma\left(E, E^{\prime}\right)}$ est faiblement complet, où $\overline{S\left(B_{E} \cap E^{+}\right)} \sigma\left(E, E^{\prime}\right)$ est l'adhérence de $S\left(B_{E} \cap E^{+}\right)$pour la topologie faible $\sigma\left(E, E^{\prime}\right)$ dans $E$. Pour cela, soit $\left(S\left(x_{i}\right)\right)_{i}$ une suite généralisée de Cauchy pour la topologie $\sigma\left(E, E^{\prime}\right)$, avec $\left(x_{i}\right)$ une suite dans $B_{E} \cap E^{+}$. Comme $S\left(B_{E} \cap E^{+}\right)$est relativement compact pour la topologie $\sigma\left(E^{\prime \prime}, E^{\prime}\right)$ dans $E^{\prime \prime}$, alors la suite $\left(S\left(x_{i}\right)\right)_{i}$ converge vers un certain $\Psi \in E^{\prime \prime}$ pour cette topologie. Soit $m \in \mathbb{N}^{*}$. D'après le Lemme 2.1, il existe $y^{m} \in E^{+}, z_{i}^{m} \in B_{E}$ et $w_{i}^{m} \in\left[0, y^{m}\right]$ tels que

$$
S\left(x_{i}\right)=\frac{1}{m} z_{i}^{m}+S\left(w_{i}^{m}\right) .
$$

D'autre part, comme $S\left(\left[0, y^{m}\right]\right)$ est faiblement compact [3, Théorème 1.2] il existe un point d'accumulation $a_{m} \in E$ de la suite généralisée $\left(w_{i}^{m}\right)_{i}$.

Fixons maintenant $f \in E^{\prime}$ avec $\|f\| \leq 1$. Alors il existe $i_{0} \in I$ tel que pour tout $i>i_{0}$, on a

Par suite,

$$
\left|f \circ S\left(x_{i}\right)-\Psi(f)\right|<\frac{1}{m} .
$$

$$
\left|f \circ S\left(w_{i}^{m}\right)-\Psi(f)\right|<\frac{2}{m} .
$$

Puisque $a_{m}$ est un point d'accumulation de la suite $\left(S\left(w_{i}^{m}\right)\right)_{i}$, il existe $i>i_{0}$ tel que

$$
\left|f \circ S\left(w_{i}^{m}\right)-\Psi\left(a_{m}\right)\right|<\frac{1}{m}
$$


ce qui donne

$$
\left|\Psi(f)-f\left(a_{m}\right)\right|<\frac{3}{m}
$$

et par suite

$$
\left\|\Psi-a_{m}\right\|_{E^{\prime \prime}} \leq \frac{3}{m}
$$

où \|\|$_{E^{\prime \prime}}$ est la norme du bidual topologique $E^{\prime \prime}$ de $E$. Par conséquent, la suite $\left(a_{m}\right)$ converge fortement vers $\Psi$, et donc $\Psi \in E$. Il s'ensuit alors que les adhérences de $S\left(B_{E} \cap E^{+}\right)$pour la topologie $\sigma\left(E, E^{\prime}\right)$ dans $E$ et dans $\hat{E}$ coïncident, où $\hat{E}$ est le completé de $E$ pour la topologie faible $\sigma\left(E, E^{\prime}\right)$. Finalement $\overline{S\left(B_{E} \cap E^{+}\right)} \sigma\left(E, E^{\prime}\right)$ est complet.

Enfin, l'implication 4) $\Longrightarrow \mathbf{1}$ ) est évidente.

Comme conséquence du Théorème 2.2 , nous obtenons le résultat suivant:

Corollaire 2.3. Soit E un treillis de Banach. Alors les assertions suivantes sont équivalentes:

1. Tout opérateur de Dunford-Pettis positif de $E$ vers $E$ est faiblement compact.

2. Pour tous opérateurs $S$ et $T$ de $E$ vers $E$ tels que $0 \leq S \leq T$ et $T$ de DunfordPettis, l'opérateur $S^{2}$ est compact.

Une autre conséquence est le résultat suivant:

Corollaire 2.4. Soit $E$ un treillis de Banach. Si la norme du dual topologique $E^{\prime}$ de $E$ est continue pour l'ordre, alors pour tous opérateurs $S$ et $T$ de $E$ vers $E$ tels que $0 \leq S \leq T$ et $T$ compact, l'opérateur $S^{2}$ est compact.

Remarque 2.5. Le corollaire 2.4 ci-dessus est un résultat d'Aliprantis et Burkinshaw [1. Théorème 2.2]. La réciproque de ce résultat a été étudiée par Wickstead dans [11, Théorème 2.2]. Il a établi que si $E$ est un treillis de Banach $\sigma$-complet pour l'ordre et si pour tous opérateurs $S$ et $T$ définis de $E$ dans $E$ vérifiant $0 \leq S \leq T$ et $T$ compact impliquent $S^{2}$ est compact, alors la norme de $E$ ou celle de $E^{\prime}$ est continue pour l'ordre. Ensuite, dans ([6, Théorème 1.1]), les deux premiers auteurs de ce papier ont étendu le résultat de Wickstead aux treillis de Banach quelconques, en montrant que si $E$ est un treillis de Banach, alors pour tous opérateurs $S$ et $T$ de $E$ dans $E$ tels que $0 \leq S \leq T$ et $T$ compact impliquent l'opérateur $S^{2}$ est compact si, et seulement si, l'une des conditions suivantes est vérifiée:

a) La norme de $E$ est continue pour l'ordre.

b) La norme de $E^{\prime}$ est continue pour l'ordre.

c) L'espace $E^{\prime}$ est discret.

Remarque 2.6. Notons que le problème de domination pour les opérateurs de Dunford-Pettis positifs sur les treillis de Banach a été étudié pour la première fois par Aliprantis et Burkinshaw dans [3]. Ils ont montré que si $E$ est un treillis de Banach qui admet une norme continue pour l'ordre et si $S, T: E \longrightarrow E$ sont deux opérateurs tels que $0 \leq S \leq T$, avec $T$ de Dunford-Pettis, alors l'opérateur $S^{2}$ est de Dunford-Pettis [3, Théorème 3.3]. Ensuite, Kalton et Saab [8, Théorème 4.4] ont établi qu'avec les mêmes hypothèses que dans [3], c'est l'opérateur $S$ qui est de Dunford-Pettis. La réciproque du résultat de Kalton et Saab a été établie par Wickstead dans [10. 
Remarque 2.7. 1. Dans [2, Théorème 7], Aliprantis et Burkinshaw ont montré que si $E$ et $F$ sont des treillis de Banach tel que la norme de $E^{\prime}$ ou celle de $F$ est continue pour l'ordre, alors pour tous opérateurs $S$ et $T$ de $E$ vers $F$ tels que $0 \leq S \leq T$ et $T$ faiblement compact, l'opérateur $S$ est faiblement compact. Dans la même année, Wickstead [9] a établi que ces conditions sont nécessaires et suffisantes (voir aussi [4, Théorème 17.10].

2. Aliprantis et Burkinshaw ont établi dans $[3$, Théorème 2.6] que si $E$ et $F$ sont des treillis de Banach tel que la norme de $F$ est continue pour l'ordre, alors pour tous opérateurs $S$ et $T$ de $E$ vers $F$ tels que $0 \leq S \leq T$ et $T$ compact impliquent $S$ est de Dunford-Pettis et faiblement compact. La réciproque de ce résultat est fausse. En effet, si $E$ est un treillis de Banach de dimension finie et $F$ un treillis de Banach de norme non continue pour l'ordre, il est clair que pour tous opérateurs $S$ et $T$ de $E$ vers $F$ tels que $0 \leq S \leq T$ et $T$ compact impliquent $S$ est de Dunford-Pettis et faiblement compact.

3. Aussi, si $E$ et $F$ sont des treillis de Banach, et si pour tous opérateurs $S$ et $T$ de $E$ vers $F$ tels que $0 \leq S \leq T$ et $T$ compact impliquent $S$ est de Dunford-Pettis et faiblement compact, alors la norme de $E^{\prime}$ n'est pas nécessairement continue pour l'ordre. En effet, il suffit de prendre $F$ un treillis de Banach de dimension finie et $E$ un treillis de Banach quelconque.

\section{RÉFÉRENCES}

[1] C. D. Aliprantis and O. Burkinshaw, Positive compact operators on Banach lattices, Math. Z., 174 (1980), 289-298. MR0593826 (81m:47053)

[2] C. D. Aliprantis and O. Burkinshaw, On weakly compact operators on Banach lattices, Proc. Amer. Math. Soc. 83 (1981), no. 3, 573-578. MR0627695 (82j:47057)

[3] C. D. Aliprantis and O. Burkinshaw, Dunford-Pettis operators on Banach lattices. Trans. Amer. Math. Soc. 274, (1982), no. 1, 227-238. MR0670929 (84b:47045)

[4] C. D. Aliprantis and O. Burkinshaw, Positive operators, Academic Press, 1985. MR0809372 (87h:47086)

[5] C.D. Aliprantis and O. Burkinshaw, Locally solid Riesz spaces with applications to economics, Mathematical Surveys and Monographs, Vol. 105, American Mathematical Society, Providence, RI, 2003. MR2011364 (2005b:46010)

[6] B. Aqzzouz and R. Nouira, Sur les opérateurs précompacts positifs. C. R. Acad. Sc. Paris, 337, (2003), no. 8, 527-530. MR2017131 (2004h:46002)

[7] Z. L. Chen and A.W. Wickstead, Vector lattices of weakly compact operators on Banach lattices, Trans. Amer. Math. Soc. 352, (2000), no. 1, 397-412. MR.1641095 (2000c:47075)

[8] N. J. Kalton and P. Saab, Ideal properties of regular operators between Banach lattices. Illinois Journal of Math. vol. 29, (1985), no. 3, 382-400. MR.0786728 (87a:47064)

[9] A. W. Wickstead, Extremal structure of cones of operators, Quart. J. Math. Oxford Ser. 2, 32 (1981), no. 2, 239-253. MR0615198 (82i:47069)

[10] A. W. Wickstead, Converses for the Dodds-Fremlin and Kalton-Saab theorems, Math. Proc. Camb. Phil. Soc. 120 (1996), no. 1, 175-179. MR1373356 (96m:47067)

[11] A.W. Wickstead, Positive compact operators on Banach lattices: some loose ends, Positivity 4 (2000), no. 3, 313-325. MR1797133(2001k:47054)

Université ibn Tofail, Faculté des Sciences, Département de Mathématiques, Equipe D'analyse Fonctionnelle, B.P. 133, Kenitra, Morocco

E-mail address: baqzzouz@hotmail.com

Université ibn Tofail, Faculté des Sciences, Département de Mathématiques, Equipe D'analyse Fonctionnelle, B.P. 133, Kenitra, Morocco

Université ibn Tofail, Faculté des Sciences, Département de Mathématiques, Equipe D’analyse Fonctionnelle, B.P. 133, Kenitra, Morocco 